\title{
SUPERCONDUCTING RF SYSTEM UPGRADE FOR SHORT BUNCH OPERATION OF CESR*
}

\author{
S. Belomestnykh ${ }^{\dagger}$, P. Barnes, R. Ehrlich, R. Geng, D. Hartill, S. Henderson, R. Kaplan, \\ J. Knobloch, H. Padamsee, S. Peck, P. Quigley, J. Reilly, D. Rubin, \\ D. Sabol, J. Sears, M. Tigner, V. Veshcherevich \\ Laboratory of Nuclear Studies, Cornell University, Ithaca, NY 14853, USA
}

\begin{abstract}
The CESR luminosity upgrade plan calls for shortening bunch length to $1 \mathrm{~cm}$. Such bunch length can be achieved by installing two more superconducting cavities to increase total RF voltage. The RF system upgrade necessary to accommodate this change is discussed.
\end{abstract}

\section{INTRODUCTION}

CESR [1] is a single-ring $e^{+} e^{-}$collider operating on and near the $Y(4 \mathrm{~S})$ resonance $(\mathrm{E} \approx 5.3 \mathrm{GeV})$. CESR has achieved peak luminosity of $1.3 \times 10^{33} \mathrm{~cm}^{-2} \mathrm{~s}^{-1}$, and total beam current of $780 \mathrm{~mA}$ in two beams. Future plans call for conversion of CESR to operate effectively over an entire range of beam energies from 1.5 to $5.6 \mathrm{GeV}$, covering the $\Psi$ and $Y$ resonances. Significant efforts will be necessary to modify CESR for low energy operation [2]. The new design, called CESRc, requires a short bunch length of $1 \mathrm{~cm}$. This translates to a total RF voltage of $12 \mathrm{MV}$ at $2.5 \mathrm{GeV}$. Two new cryomodules have been ordered in addition to the existing four to provide voltage necessary for bunch shortening. Six cavities will deliver $12 \mathrm{MV}$ with confidence, operating at the average accelerating gradient of $6.7 \mathrm{MV} / \mathrm{m}$. Further increase of RF voltage up to $19 \mathrm{MV}$ is possible by lowering operating temperature of cavities to $3 \mathrm{~K}$.

\section{PRESENT RF ACCELERATING SYSTEM}

CESR RF system [3] consists of four single-cell superconducting cavity cryomodules, operating at an average gradient of $6.2 \mathrm{MV} / \mathrm{m}$, providing a total voltage of 7.4 MV and supplying RF power in excess of $280 \mathrm{~kW}$ per cavity to high current beams (Figure 1). The cryomodules are installed in pairs in the East (E1 and E2 locations) and in the West (W1 and W2) RF straight sections in the CESR tunnel. Cryogen liquids are supplied to cryomodules via satellite distribution boxes located next to the cavities in the tunnel. A fifth, spare cryomodule reached accelerating gradient of $8 \mathrm{MV} / \mathrm{m}$ in $\mathrm{CW}$ and $14.5 \mathrm{MV} / \mathrm{m}$ in pulsed mode during high power test.

Four transmitters are available to provide RF power to the cavities in CESR and to the RF processing area to

\footnotetext{
*Work supported by the National Science Foundation.

${ }^{\dagger}$ sab@lns.cornell.edu
}

perform various high power tests. Each transmitter uses a 600 kWCW YK1300 klystron. The system can be configured to provide RF power from one klystron to one or two cavities depending on RF power demand. The RF power distribution system consists of WR1800 waveguide straight sections, bends, and magic T's and ferrite circulators. At present, due to both RF power demand and desire to have more flexibility to adjust RF parameters of individual cavities, the RF system configuration is one cavity per klystron.

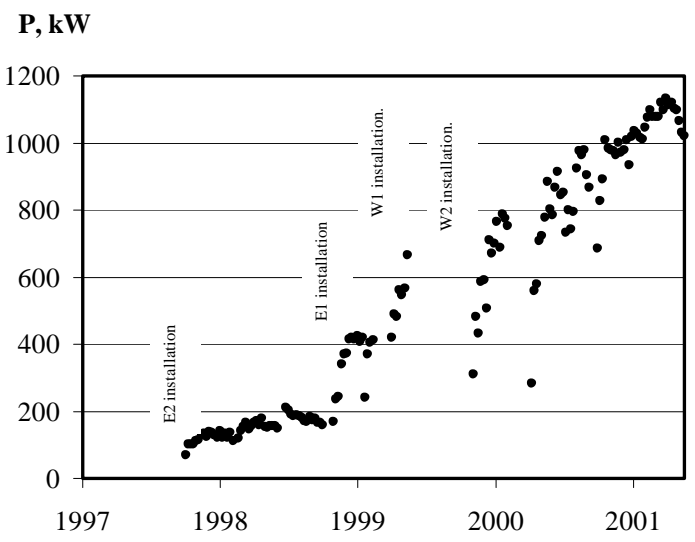

Figure 1: Weekly maximum RF power delivered to CESR beams by superconducting cavities.

\section{UPGRADE FOR LOW ENERGY OPERATION}

\subsection{Hardware upgrade}

CESRc design calls for a short bunch length of $1 \mathrm{~cm}$ and requires an RF voltage increase (see Table). Some additional voltage is available by raising operating gradient of existing cavities. Two of the cavities, E2 and $\mathrm{W} 1$, can operate at the accelerating gradient of $8 \mathrm{MV} / \mathrm{m}$ producing 2.4 MV per cavity. Two others, E1 and W2, are gradient limited by surface defects to $6.5-7 \mathrm{MV} / \mathrm{m}$ [3]. We are confident that E1 cavity can be repaired and its performance improved. Repair of W2 cavity is more problematic due to the nature of the defects. Tests of this cavity indicate that it has multiple defects embedded into the material during manufacturing process of the niobium sheets, which will be very difficult, if possible at all, to remove. W2 will have to be replaced with a healthier 
spare cavity. Thus, after repairing the E1 cavity and replacing the W2 with spare cryomodule we could have four cavities capable to operate at $8 \mathrm{MV} / \mathrm{m}$. This will provide CESR with maximum RF voltage of $9.6 \mathrm{MV}$, which is $2.4 \mathrm{MV}$ short of the required $12 \mathrm{MV}$. Therefore two new cryomodules have been ordered to provide additional RF voltage. Maximum average accelerating gradient with six cavities in the ring will be $6.7 \mathrm{MV} / \mathrm{m}$. In case if CESRc lattice change will call for even higher RF voltage, it is possible to increase it by raising accelerating gradient to $8 \mathrm{MV} / \mathrm{m}$ at $4.5 \mathrm{~K}$ reaching $14.4 \mathrm{MV}$. Further increase would require improving $Q$ factors of all cavities by means of vacuum bake at $140^{\circ} \mathrm{C}$ [3] and lowering operating temperature of the two new cavities to $3 \mathrm{~K}$. Then we should be able to operate four old cavities at 3 MV per cavity (accelerating gradient of $10 \mathrm{MV} / \mathrm{m}$ at 4.5 $\mathrm{K}$ ) and two new ones at $3.5 \mathrm{MV}$ per cavity (at $3 \mathrm{~K}$ ), providing total RF voltage of $19 \mathrm{MV}$.

Table: Selected parameters of CESR

\begin{tabular}{|l|c|c|c|c|}
\hline Parameter & Present & \multicolumn{3}{|c|}{ CESRc upgrade } \\
\hline Energy [GeV] & 5.3 & 1.55 & 1.89 & 2.5 \\
\hline No. of cavities & 4 & 6 & 6 & 6 \\
Gradient [MV/m] & 6.2 & 4.2 & 5.6 & 6.7 \\
Voltage [MV] & 7.4 & 7.5 & 10 & 12 \\
Beam power [MW] & 1.1 & 0.04 & 0.09 & 0.16 \\
Beam current [A] & 0.75 & 0.26 & 0.36 & 0.46 \\
Bunch length [mm] & 18 & 9.9 & 10.2 & 10.2 \\
\hline
\end{tabular}

New cryomodules will be located in the CESR interaction region (IR) on both sides of the detector CLEO. Two satellite cold valve boxes will be installed in the IR region to provide cryogenic coolants to new cavities. Their design will be similar to the design of valve boxes used for existing cryomodules but each will feed one cavity instead of two.

High power part of RF transmitters will not require significant modifications. New waveguide feeder will be added to IR cavities for high power processing and supplying RF power in active mode of operation. Threestub waveguide transformers [4] will be installed to individually adjust cavity couplings to their optimal values. Three transmitters will be configured to two cavities per klystron. The forth transmitter will be available for processing area tests.

New RF and cryogenic controls, data acquisition electronics, and interlocks will be designed and built for IR cavities. This will provide a basis for possible future upgrade of electronics for other cavities.

\subsection{Passive mode of cavity operation}

While RF voltage required by CESRc is high, RF power demand is very moderate. These power levels hardly present a problem for RF power couplers of CESR superconducting cavities. Even one transmitter is more than adequate to supply necessary power. On the other hand, such a low power demand at very high voltage will present a problem for RF regulation loops [5]. To ease this problem we propose to operate two out of six cavities in active mode and the other four in passive mode. The function of the four passive cavities will be to provide missing voltage to shorten bunches. This voltage is induced by the beam and therefore its phase follows the beam automatically. But there are some difficulties associated with this mode of operation.

Let us define generalized detuning [6] as

$$
\xi=Q_{l}\left(\frac{\omega_{R F}}{\omega}-\frac{\omega}{\omega_{R F}}\right)=-\tan \psi \approx 2 Q_{l} \frac{\Delta \omega}{\omega_{R F}},
$$

where $Q_{l}$ is the cavity loaded quality factor (it determines the amount of power extracted from the beam), $\omega_{\mathrm{RF}}$ is the $\mathrm{RF}$ frequency, $\omega$ is the cavity resonance frequency, $\Delta \omega=$ $\omega_{\mathrm{RF}}-\omega$, and $\psi$ is the cavity tuning angle. $\xi$ measures the amount of cavity detuning from the RF frequency in units of one half of a cavity bandwidth. Initially cavity is parked at a "home" position $\xi_{0}$. Then the beam-induced voltage is changing linearly with the beam current:

$$
V(I)=\frac{I \cdot R / Q \cdot Q_{l}}{\sqrt{1+\xi_{0}^{2}}} \approx I \cdot R / Q \cdot \frac{1}{2} \frac{\omega_{R F}}{\Delta \omega_{0}},
$$

where $I$ is the beam current and $R / Q$ is the cavity shunt impedance. The approximate formula is valid when cavity is detuned far enough from resonance $\left(\xi_{0}>>1\right)$, which is usually true. As soon as the cavity voltage reaches a certain level (say $V_{0}=1.67 \mathrm{MV}$ for $E=1.89$ $\mathrm{GeV}$ ), the tuner feedback loop begins to operate to keep the voltage constant by detuning the cavity according to

$$
\Delta \omega(I)=\omega_{R F} \cdot \frac{I \cdot R / Q}{2 V_{0}}=\Delta \omega_{0} \frac{I}{I_{0}} .
$$

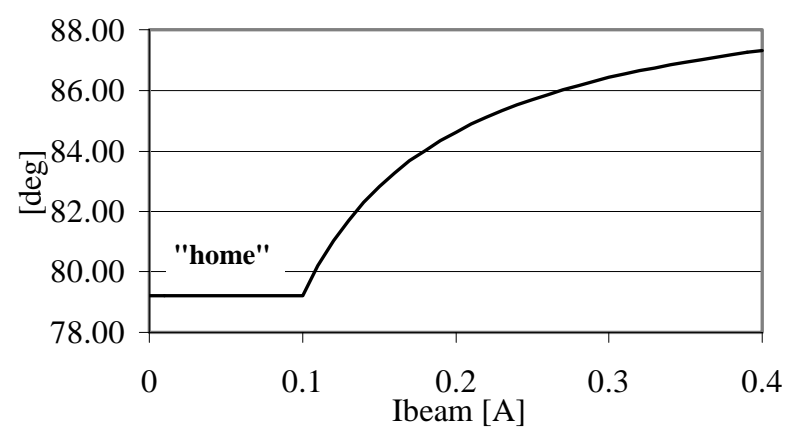

Figure 2: Tuning angle dependence on beam current.

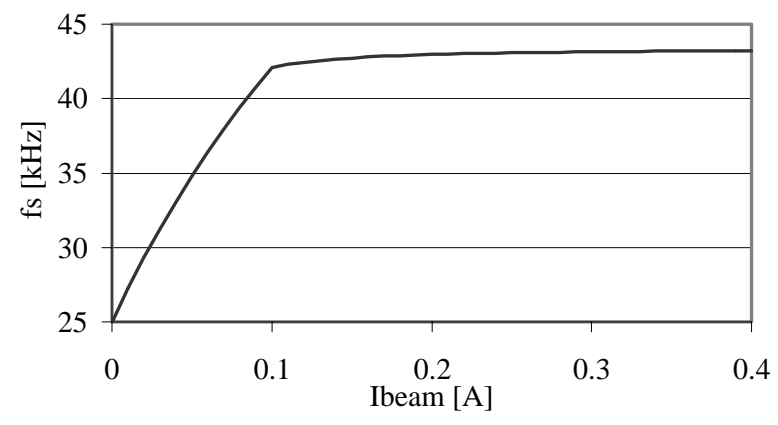

Figure 3: Synchrotron tune change with beam current. 


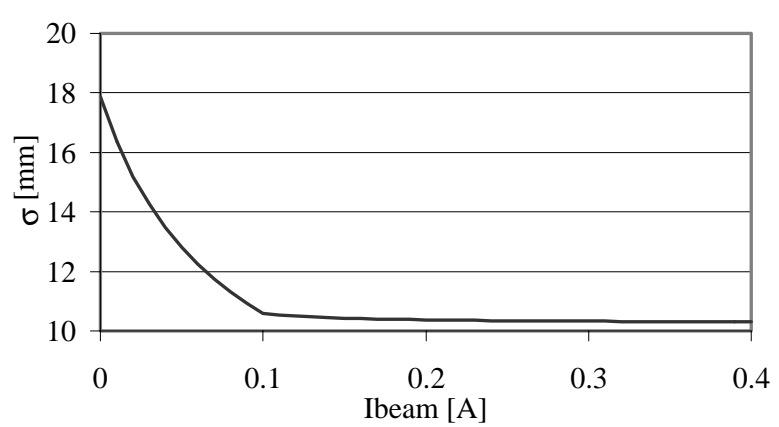

Figure 4: Bunch length dependence on beam current.

Figure 2 illustrates the change of the tuning angle with the beam current. The "home" position determines the beam current $I_{0}$ at which cavity voltage reaches its operating level $V_{0}$ :

$$
I_{0}=2 \frac{\Delta \omega_{0}}{\omega_{R F}} \frac{V_{0}}{R / Q} .
$$

The synchrotron tune and bunch length will be changing with the beam current as well (Figures 3 and 4). The coherent tune shift [7] is negligibly small for the considered example. Special arrangements will be needed to assure proper operation of the longitudinal feedback system.

To check feasibility of the passive mode of operation we performed a machine studies experiment. A tuner control loop of one of the four SRF cavities installed in CESR was reconfigured for the passive regime. The passive cavity home position was set so that it would reach the voltage set point at a beam current of $100 \mathrm{~mA}$. We monitored the beam induced voltage and the synchrotron tune. Figure 5 presents measured synchrotron frequency in comparison with calculations. Plots show that measured data points deviate from the predicted curve if one does not take into account coherent tune shift in this case. Our experiment confirmed feasibility of the passive mode operation of superconducting cavities in CESR.

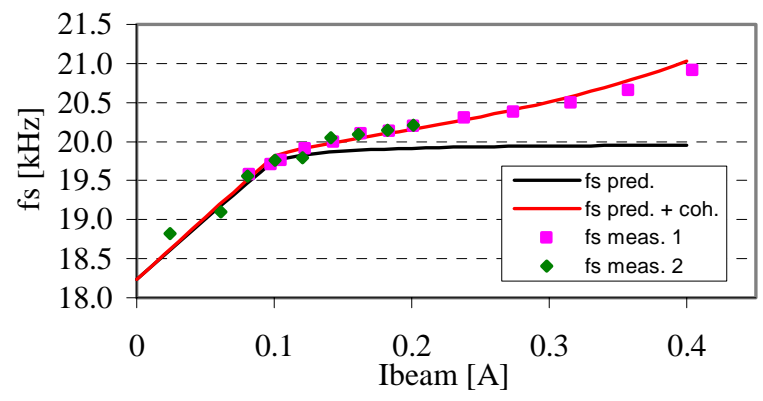

Figure 5: Comparison of measured synchrotron frequency with predictions.

\subsection{Amplitude and phase modulation of passive cavity voltage caused by microphonics}

Microphonic noise due to mechanical vibrations and cryogen liquid turbulence causes cavity resonance to wander around. This produces the amplitude and the phase modulation of the passive cavity voltage. The formulae for these modulations are:

$$
\begin{aligned}
& \frac{\delta V}{V_{0}}=\frac{\delta \omega}{\Delta \omega_{0}} \frac{I_{0}}{I}, \\
& \delta \psi=\frac{\delta \omega}{\Delta \omega_{0}} \frac{1}{\xi_{0}}\left(\frac{I_{0}}{I}\right)^{2} .
\end{aligned}
$$

CESR data indicate that parasitic change of the cavity resonant frequency $\delta \omega$ is of the order of $2 \pi \times 25 \mathrm{~Hz}$. Then for $V_{0}=1.67 \mathrm{MV}, I_{0}=100 \mathrm{~mA}$, and $Q_{l}=1 \times 10^{6}$ one can calculate $\delta V / V_{0}=1.5 \times 10^{-3}$ and $\delta \psi=0.0044^{\circ}$ for beam current of $360 \mathrm{~mA}$. This modulation is much smaller than typically observed in CESR and will not cause any degradation of the RF system performance [5].

\section{CONCLUSIONS}

We plan to upgrade the superconducting RF system for short bunch operation of CESR. Addition of two new cryomodules will allow the RF system to provide total accelerating voltage of $12 \mathrm{MV}$ to shorten bunch length in the storage ring to $1 \mathrm{~cm}$. Further increase of RF voltage up to $19 \mathrm{MV}$ is possible as well by lowering operating temperature from $4.5 \mathrm{~K}$ to $3 \mathrm{~K}$.

Very tight requirements to RF regulation loops and low power demand at low energy lead us to propose operating four out of six cavities in the passive mode. Results of the calculations and the experiment indicate that this mode of operation is feasible.

\section{REFERENCES}

[1] D. Rubin, et al., "CESR Status," this conference.

[2] D. Rice, et al., "Parameters for Low Energy Operation of CESR," this conference.

[3] S. Belomestnykh, et al., "Running CESR at High Luminosity and Beam Current with Superconducting RF System," Proc. of the 7th EPAC, Vienna, Austria, June 2000, Vol. 3, pp. 2025-2027.

[4] B. Dwersteg, "SC-Cavity Operation via WGTransformer." Proceedings of the 4th SRF Workshop, KEK Report 89-21, Vol. 2, pp. 593-604.

[5] S. Belomestnykh, et al., "Superconducting RF Control Issues at CESR," this conference.

[6] I. S. Gonorovsky, Radio Circuits and Signals, Radio I Svyaz', Moscow (1986), in Russian.

[7] A. Chao, Physics of Collective Beam Instabilities in High Energy Accelerators, Wiley (1993). 